\title{
Molecular Diagnosis and Translymphatic Chemotherapy Targeting Sentinel Lymph Nodes of Patients with Early Gastrointestinal Cancers
}

\author{
Hiroya Takeuchi Masakazu Ueda Takashi Oyama Yoshimasa Shimizu \\ Yuko Kitagawa
}

Department of Surgery, Keio University School of Medicine, Tokyo, Japan

\section{Key Words}

Sentinel lymph node - Micrometastasis • RT-PCR •

Paclitaxel $\cdot 2$-Methacryloyloxyethyl phosphorylcholine

\begin{abstract}
The sentinel lymph node (SLN) is defined as the lymph node(s) that first receives lymphatic drainage from the site of the primary tumor. Recent progress in molecular techniques has demonstrated the presence of micrometastatic tumor cells in SLNs. Quantitative real-time RT-PCR assay, which enables rapid analysis, is currently being undertaken for intraoperative molecular diagnosis of SLNs. We developed an intraoperative real-time RT-PCR assay to detect micrometastasis in SLNs for early gastric cancer. All SLNs and randomly selected nonSLNs in 96 cT1 or CT2 gastric cancer patients were biopsied intraoperatively and examined by routine hematoxylin and eosin staining, immunohistochemistry with anticytokeratin antibody (AE1/AE3), and multimarker real-time RT-PCR assay including cytokeratin (CK) 19, CK20, and carcinoembryonic antigen. All patients with histopathologically verified metastasis in their SLNs demonstrated positive results by RT-PCR assay. Forty percent of patients with histopathologically negative SLNs showed positive SLNs by RT-PCR assay. RT-PCR assay revealed that 4 patients (4\%) with negative SLNs had positive non-SLNs; however, these positive non-SLNs were identified within each SLN basin. We recently developed a new drug delivery system targeting SLNs with a phospholipid polymer, using 2-methacryloyloxyethyl phosphorylcholine
\end{abstract}

conjugated with paclitaxel. Our preliminary data suggest that this novel drug delivery system may be feasible for translymphatic chemotherapy targeting SLNs of patients with cNO early gastrointestinal cancer, who have the potential for occult metastasis in SLNs. Endoscopic resection of the primary tumor followed by translymphatic chemotherapy targeting SLNs may become a promising minimally invasive multidisciplinary therapy.

Copyright $\odot 2010$ S. Karger AG, Basel

\section{Introduction}

The sentinel lymph node (SLN) is defined as the lymph node(s) that first receives lymphatic drainage from the primary site of a tumor [1]. SLN is thought to be the first possible site of micrometastasis along the route of lymphatic drainage from the primary lesion. Thus, the pathological status of SLN can predict the status of all regional lymph nodes. If SLN is recognizable and negative for cancer metastasis, unnecessary radical lymph node dissection may be avoided.

This 'SLN concept' developed by Morton et al. [1] is well established in the treatment of patients with several types of solid carcinomas, such as melanoma and breast cancer [2-4]. The concept allows a more focused and efficient pathological analysis of micrometastatic disease in regional lymph nodes. The SLN concept has revolutionized the approach to the surgical staging of both mela-

\section{KARGER}

Fax +4161306 1234 E-Mail karger@karger.ch www.karger.com

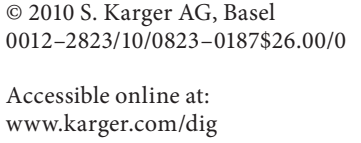

Hiroya Takeuchi, $\mathrm{MD}, \mathrm{PhD}$

Department of Surgery, Keio University School of Medicine

35 Shinanomachi, Shinjuku-ku

Tokyo 160-8582 (Japan)

Tel. +81 33353 1211, Fax +81 33355 4707, E-Mail htakeuch@ @c.itc.keio.ac.jp 
noma and breast cancer, and these techniques can benefit patients by preventing various complications due to unnecessary prophylactic radical lymphadenectomy when SLN is negative for cancer metastasis. More recently, the SLN concept was extended to many other solid tumors, including gastrointestinal (GI) cancers [5-8]. Diagnostic accuracy of micrometastasis is the minimum requirement for the clinical application of SLN mapping for early GI cancer. In this work, we review the clinical significance of molecular diagnosis for micrometastasis of SLN in gastric cancer and also consider a newly developed translymphatic chemotherapy targeting SLN.

\section{Molecular Diagnosis of SLN in Gastric Cancer}

In general, hematoxylin and eosin (HE) staining as well as immunohistochemical (IHC) staining have been commonly used in combination with serial sectioning of frozen and paraffin-embedded specimens for the detection of micrometastatic disease in SLN [9-11]. Application of IHC has markedly improved the sensitivity of micrometastatic disease detection in SLN, beyond the capability of routine HE staining alone. However, even IHC has its limitations in terms of sensitivity and specificity. Antibodies against tumor markers of interest must be highly specific and sensitive for detection of tumor cells but virtually non-reactive to adjacent non-tumor cells in SLN.

Molecular detection of tumor cells using RNA or DNA markers with various PCR techniques has evolved exponentially in the last decade. The primary approach to the molecular detection of tumor cells has focused on mRNA of tumor markers, using RT-PCR assay. Detection of metastatic tumor cells has been clearly demonstrated in lymph nodes. Using RT-PCR, it is now possible to reliably detect 1-10 tumor cells on a background of $10^{6}-10^{7}$ normal cells [12]. The high sensitivity of RT-PCR assay compared to $\mathrm{HE}$ and IHC staining enables accurate detection of occult tumor cells among the lymphoid cells present in SLNs [13]. However, particular attention must be paid to technique through all stages of the assay in order to ensure accurate results. One of the keys to high efficiency in RTPCR assay is the quality of the detection marker. Finding a good marker is of utmost importance in molecular detection. We previously reported that $20 \%$ of patients with GI cancers were upstaged by RT-PCR assay using CK19 in SLNs [14].

Quantitative real-time RT-PCR assay is now being used more extensively not only to detect the presence of target mRNA but also to quantify the number of mRNA copies from tumor-associated genes. Quantitative real-time RTPCR analysis permits rapid molecular analysis of multiple mRNA targets expressed in tumor cells, and the results can then be correlated with clinical outcomes in order to study the relationship between gene expression levels and outcome [15]. The real-time RT-PCR assay, which enables rapid analysis, is currently being undertaken for intraoperative molecular diagnosis of SLNs. SLNs obtained from patients have been assessed at some institutions by intraoperative histopathological examination and real-time RT-PCR assay using multiple markers [13].

SLN mapping in open and laparoscopic surgery for gastric cancer has been attempted in our and other institutions [7]. Reports of SLN mapping for early gastric cancer demonstrate almost acceptable results in terms of the detection rate and accuracy in determining lymph node status. Appropriate indications for proximal gastrectomy, segmental gastrectomy, pylorus-preserving gastrectomy, and partial resection for $\mathrm{CT} 1 \mathrm{~N} 0$ gastric cancer were individually determined based on the distribution of SLN basins and the metastatic status of SLNs [7]. However, the sensitivity of intraoperative diagnosis of micrometastasis is a crucial part of SLN mapping in gastric cancer. Arigami et al. [16] reported that 13 of 53 (25\%) gastric cancer patients with histopathologically negative SLNs were upstaged by RT-PCR assay. They concluded that the SLN concept is applicable to patients with cT1N0 gastric cancer, even when including the molecular diagnosis of micrometastasis.

We also recently developed an intraoperative realtime RT-PCR assay to detect micrometastasis in SLNs for early gastric cancer. The operation time from RNA extraction until obtaining the results is about $60-90 \mathrm{~min}$. In our preliminary study, SLNs were identified by dualtracer methods using a radioactive tracer and blue dye in 96 cT1 or cT2 gastric cancer patients [7]. Histopathological examination (HE and IHC) and intraoperative realtime RT-PCR assay were performed for 435 SLNs and 304 non-SLNs that were randomly selected. All SLNs and non-SLNs were biopsied and bivalved intraoperatively and examined by routine HE and IHC staining with anticytokeratin antibody (AE1/AE3) and multimarker realtime RT-PCR assay including cytokeratin (CK) 19, CK20, and carcinoembryonic antigen. All 18 patients with histopathologically verified metastasis in their SLNs demonstrated positive results in the RT-PCR assay. Thirtyeight $(40 \%)$ patients with histopathologically negative SLNs showed positive SLNs in the RT-PCR assay. Four patients (4\%) had negative SLNs but positive non-SLNs in the RT-PCR assay. Thus, molecular diagnosis gave false- 


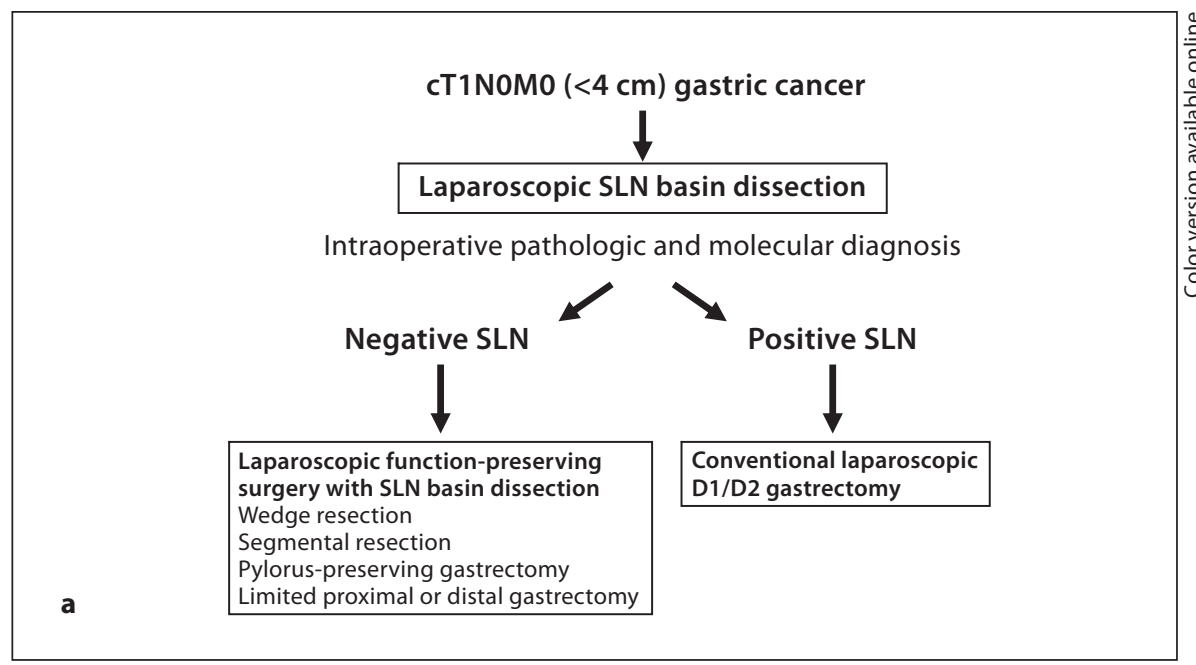

Fig. 1. a Laparoscopic function-preserving gastrectomy with sentinel lymphatic basin dissection based on molecular diagnosis of SLNs. b Current approaches for cT1N0M0 gastric cancer based on SLN navigation.

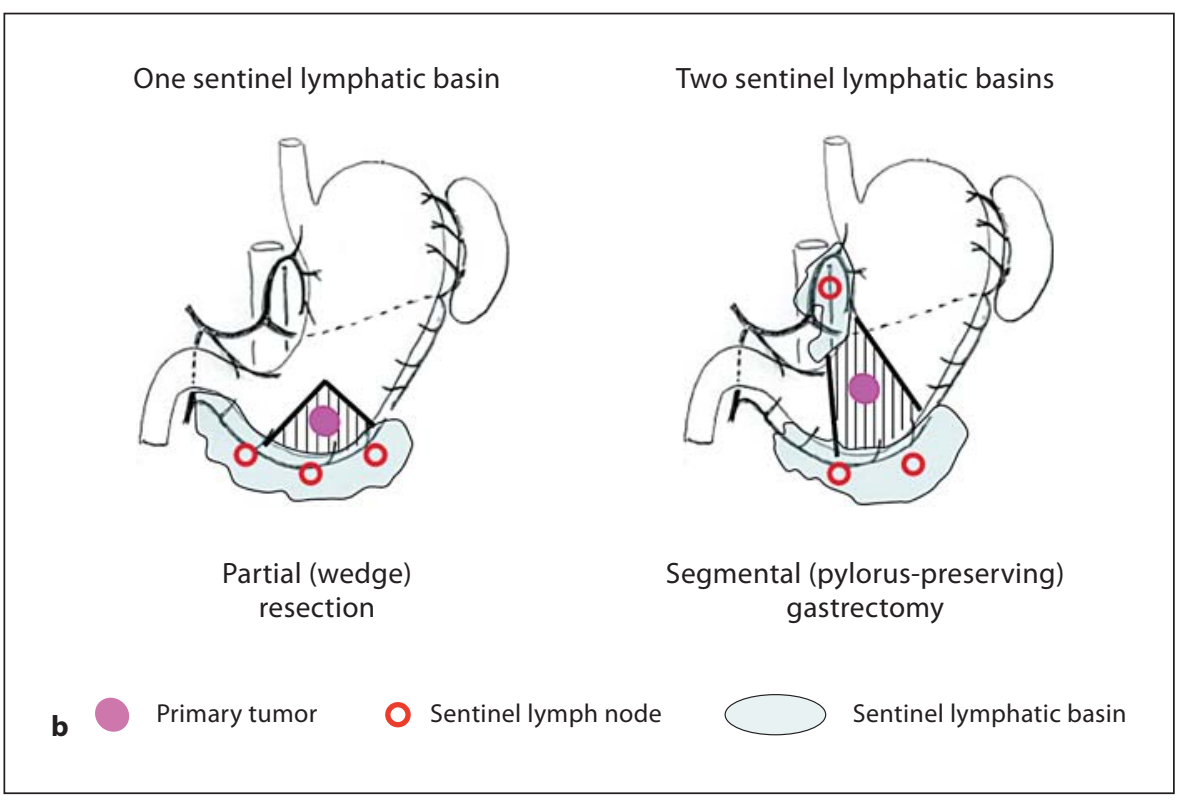

negative results, however these non-SLNs in the 4 falsenegative cases were identified within each SLN basin.

In conclusion, we verified the reliability of the newly developed intraoperative RT-PCR assay in patients with early gastric cancer. The results suggest that molecular diagnosis by our RT-PCR method may prove the SLN concept for early gastric cancer, and SLN basin dissection may provide us with an acceptable safety net for clinical application. At present, surgical treatment of cT1N0 gastric cancer can be individualized, as shown in the flowchart based on SLN navigation (fig. 1a) [7]. Various types of laparoscopic function-preserving surgery are applicable in cases with cancer-negative SLN determined by in- traoperative pathological and molecular diagnosis. For example, laparoscopic partial (wedge) resection of the stomach is applicable in cases with one sentinel lymphatic basin on the side of the greater curvature (fig. 1b). On the other hand, laparoscopic segmental gastrectomy with a pylorus-preserving procedure is feasible for cases in which the primary lesion is $>4 \mathrm{~cm}$ from the pylorus ring and there are two sentinel lymphatic basins on the lesser and greater curvature sides (fig. 1b). Earlier recovery after surgery and preservation of quality of life in the late phase may be achieved by laparoscopic limited gastrectomy with SLN navigation and intraoperative molecular diagnosis of SLN. 
Fig. 2. a The experimental metastasis model of mesocecal SLNs. SLN in the model is defined as the blue node(s) located within mesocecum, which is immediately and reproducibly stained after injection of isosulfan blue into the cecal submucosa. \#2 = Secondary node; \#3 = distant node. $\mathbf{b}$ Weight of metastatic SLNs in the group treated with PTX/PMB30W conjugate compared to the group without treatment.
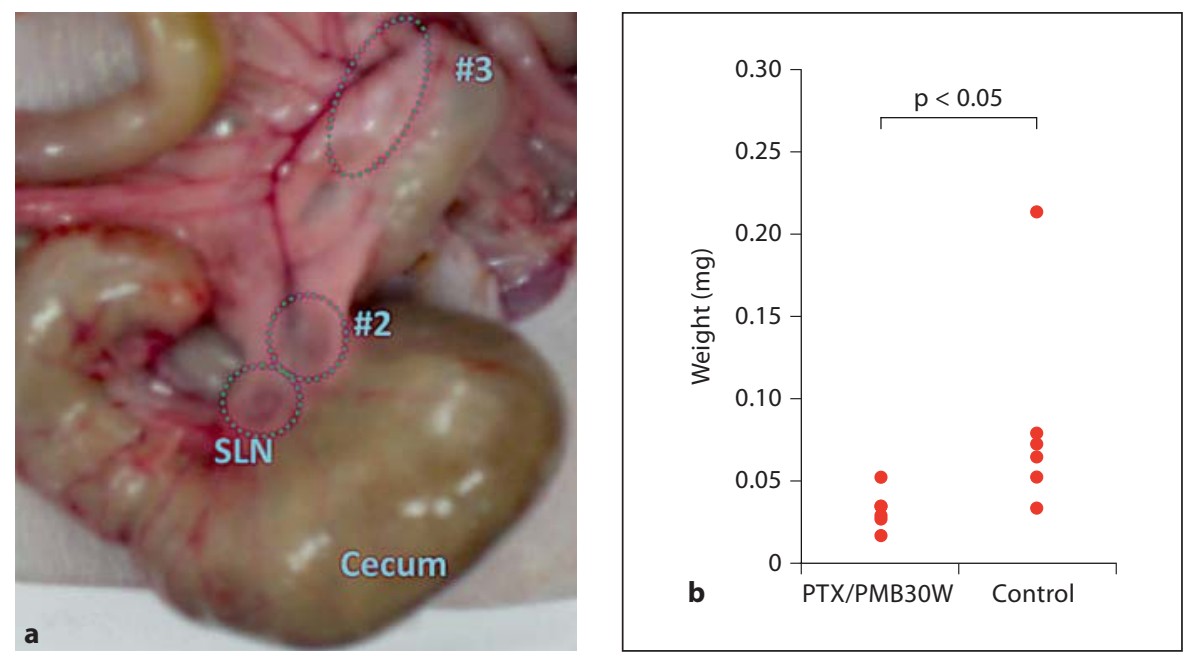

Translymphatic Chemotherapy Targeting SLN Using a Novel Drug Delivery System

We recently developed a drug delivery system targeting SLNs with a novel phospholipid polymer, using 2-methacryloyloxyethyl phosphorylcholine (MPC) conjugated with paclitaxel (PTX). The MPC polymer has the same polar phosphorylcholine group as that found in biomembranes, which is likely to inhibit non-specific interactions with biomaterials, including proteins and cells [17-19]. The MPC unit can be copolymerized with any vinyl monomer by a conventional radical polymerization technique. The most effective phospholipid polymer had a hydrophilic MPC unit and $70 \mathrm{~mol} \%$ of a hydrophobic butyl methacrylate (BMA) unit as the co-monomer. This poly(MPC-co-BMA), referred to as PMB30W, forms a polymer aggregate in water through hydrophobic interactions but still has excellent water solubility. In the aqueous in vivo environment, MPC will face outward in contact with the solvent, whereas the BMA units will tend to aggregate in an inner layer.

PMB30W forms stable polymeric lipid nanoparticles that completely dissolve a large amount of PTX without the need for specific additives. PTX is a promising drug for the treatment of various types of cancers, including those of the breast, ovary, head and neck, stomach, and lung. However, because of its low water solubility, special solvents are required to dissolve it. Local administration of these solvents is known to cause various clinical problems, such as skin or mucosal necrosis and allergic reactions. On the other hand, we previously reported that PMB30W alone did not show any toxicity or severe side effects nor did subcutaneous injection of PTX/PMB30W damage the skin [17-19]. Around $10 \%$ of PTX was released continuously from the PTX/PMB30W conjugate in the first $24 \mathrm{~h}$. In addition, the particle diameter was estimated to be about $50 \mathrm{~nm}$, which is the optimal size of a tracer for SLN mapping.

We hypothesized that submucosal injection of PTX/ PMB30W around the primary GI cancer lesion results in translymphatic accumulation of PTX, sufficient to control the subclinical metastatic tumor in SLNs with no local reaction at the injection site. The experimental metastasis model using mesocecal lymph nodes established by Nagata et al. [20] corresponds well with metastasis to SLNs in GI cancers. In brief, male Donryu rats weighing 200-320 g were used in the study. The rats were anesthetized and the abdomen was opened with a small midline incision. Approximately $0.05 \mathrm{ml}$ of cancer cell suspension (AH130; $5 \times 10^{5}$ cells) was inoculated into the cecal submucosa using a microsyringe with a 30 -gauge needle. SLN in the model is defined as the blue node(s) located within the mesocecum, which is immediately and reproducibly stained after injection of isosulfan blue into the cecal submucosa (fig. 2a). Several days after tumor inoculation, proliferated cancer cells were visualized in the SLNs.

In our preliminary study, PTX/PMB30W conjugate solution (concentration of PTX, $5 \mathrm{mg} / \mathrm{ml}$ ) was directly injected into the cecal submucosa in the same manner on the day after tumor inoculation. A high concentration of PTX in SLNs was maintained for at least $24 \mathrm{~h}$ after submucosal injection of PTX/PMB30W (data not shown). On day 7 after tumor inoculation, not only the primary tumor but also the metastatic SLNs in the group treated 
with the conjugate solution were markedly smaller than those in the group not given conjugate treatment (fig. 2b). Injection of PTX/PMB30W solution into the cecal submucosa caused no adverse local reactions in the cecum, such as erosions, ulcers, or necrosis.

Further preclinical and clinical studies will be required to verify the efficacy and safety of the PTX/PMB30W conjugate solution in clinical practice. However, our results suggest that the new drug delivery system may be feasible for local chemotherapy targeting SLNs in patients with cN0 early GI cancer, in whom there is potential for metastasis in SLNs. Endoscopic resection of the primary tumor followed by selective chemotherapy targeting SLNs may be a promising minimally invasive multidisciplinary therapy.

\section{Conclusion}

Development of the SLN concept has radically altered the field of diagnosis and treatment of many solid tumors. Increasing the sensitivity of occult tumor cell detection in
SLN using molecular-based analysis provides a more accurate understanding of the clinical significance of micrometastatic nodal disease. Moreover, molecular staging of SLNs should benefit and improve the surgical management of patients with early GI cancers. Individualized minimally invasive GI surgery, such as laparoscopic function-preserving gastrectomy, should be verified as curative and beneficial for patients with cancer-negative SLNs, as determined by intraoperative molecular diagnosis.

In addition, selective chemotherapy targeting SLN appears attractive for patients with early GI cancer who may have subclinical occult SLN metastasis. Further investigations may lead to the development of a new minimally invasive multimodal therapy targeting both the primary tumor and SLNs in the near future.

\section{Disclosure Statement}

This study was partly supported by the Molecular Diagnostics R\&D Department, Roche Diagnostics KK, Tokyo, Japan.

\section{References}

1 Morton DL, Wen DR, Wong JH, et al: Technical details of intraoperative lymphatic mapping for early stage melanoma. Arch Surg 1992;127:392-399.

-2 Giuliano AE, Kirgan DM, Guenther JM, Morton DL: Lymphatic mapping and sentinel lymphadenectomy for breast cancer. Ann Surg 1994;220:391-401.

$\checkmark 3$ Morton DL, Thompson JF, Essner R, Elashoff R, Stern SL, Nieweg OE: Validation of the accuracy of intraoperative lymphatic mapping and sentinel lymphadenectomy for early-stage melanoma: a multicenter trial. Multicenter Selective Lymphadenectomy Trial Group. Ann Surg 1999;230:453463.

4 Krag D, Weaver D, Ashikaga T, et al: The sentinel node in breast cancer - a multicenter validation study. N Engl J Med 1998;339: 941-946.

5 Bilchik AJ, Saha S, Wiese D, et al: Molecular staging of early colon cancer on the basis of sentinel node analysis: a multicenter phase II trial. J Clin Oncol 2001;19:1128-1136.

-6 Kitagawa Y, Fujii H, Mukai M, et al: The role of the sentinel lymph node in gastrointestinal cancer. Surg Clin North Am 2000;80: 1799-1809.

77 Takeuchi H, Saikawa Y, Kitagawa Y: Laparoscopic sentinel node navigation surgery for early gastric cancer. Asian J Endosc Surg 2009;2:13-17.
8 Takeuchi H, Fujii H, Ando N, et al: Validation study of radio-guided sentinel lymph node navigation in esophageal cancer. Ann Surg 2009;249:757-763.

$\checkmark 9$ Cote RJ, Peterson HF, Chaiwun B, et al: Role of immunohistochemical detection of lymph-node metastases in management of breast cancer. International Breast Cancer Study Group. Lancet 1999;354:896-900.

10 Czerniecki BJ, Scheff AM, Callans LS, et al: Immunohistochemistry with pancytokeratins improves the sensitivity of sentinel lymph node biopsy in patients with breast carcinoma. Cancer 1999;85:1098-1103.

-11 Turner RR, Ollila DW, Krasne DL, Giuliano AE: Histopathologic validation of the sentinel lymph node hypothesis for breast carcinoma. Ann Surg 1997;226:271-276.

12 Pantel K, Cote RJ, Fodstad O: Detection and clinical importance of micrometastatic disease. J Natl Cancer Inst 1999;91:11131124.

13 Takeuchi H, Kitajima M, Kitagawa Y: Sentinel lymph node as a target of molecular diagnosis of lymphatic micrometastasis and local immunoresponse to malignant cells. Cancer Sci 2008;99:441-450.

14 Matsuda J, Kitagawa Y, Fujii H, et al: Significance of metastasis detected by molecular techniques in sentinel nodes of patients with gastrointestinal cancers. Ann Surg Oncol 2004; 11:250S-254S
15 Takeuchi H, Kuo C, Morton DL, Wang HJ, Hoon DS: Expression of differentiation melanoma-associated antigen gene is associated with favorable disease outcome in advancedstage melanomas. Cancer Res 2003;15:441448 .

16 Arigami T, Natsugoe S, Uenosono Y, et al: Evaluation of sentinel node concept in gastric cancer based on lymph node micrometastasis determined by reverse transcription-polymerase chain reaction. Ann Surg 2006;243:341-347

17 Chiba N, Ueda M, Shimada T, et al: Novel immunosuppressant agents targeting activated lymphocytes by biocompatible MPC polymer conjugated with interleukin-2. Eur Surg Res 2007;39:103-110.

$\checkmark 18$ Wada M, Jinno H, Ueda M, et al: Efficacy of an MPC-BMA co-polymer as a nanotransporter for paclitaxel. Anticancer Res 2007;27: 1431-1435.

19 Shimada T, Ueda M, Jinno H, et al: Development of targeted therapy with paclitaxel incorporated into EGF-conjugated nanoparticles. Anticancer Res 2009;29:1009-1014.

20 Nagata H, Arai T, Soejima Y, et al: Limited capacity of regional lymph nodes to eradicate metastatic cancer cells. Cancer Res 2004;64:8239-8248. 